\title{
A survey of the knowledge and practices of nursing students of Mbarara University of Science and Technology around Monitoring Fluid Requirements for burns patients on surgical ward at Mbarara Regional Referral Hospital.
}

Joan Atuhaire

Mbarara University of Science and Technology

Jonathan Kajjimu ( $\nabla$ jonathkebenz37@gmail.com )

Mbarara University of Science and Technology

Grifance Opio

Mbarara University of Science and Technology

Frank Lubega

Mbarara University of Science and Technology

Reagan Kakande

Mbarara University of Science and Technology

William Mwanje

Mbarara University of Science and Technology

Andrew Tagg

Western Health

\section{Research Article}

Keywords: fluid balance, fluid monitoring, nursing students, burns, Uganda

Posted Date: January 26th, 2022

DOI: https://doi.org/10.21203/rs.3.rs-1298862/v1

License: (c) (i) This work is licensed under a Creative Commons Attribution 4.0 International License.

Read Full License 


\section{Abstract}

Background There is a high mortality of burns especially in low- and middle-income countries which already have less developed healthcare systems. Besides, little is known about nursing students' knowledge and practices towards the need to monitor fluid requirement in admitted burns patients.

Objective To assess the knowledge and practices of nursing students regarding monitoring fluid requirements for hospitalised paediatrics and adult burn patients on the surgical ward at Mbarara regional referral hospital.

Methods We conducted an online descriptive cross-sectional study among clinical nursing students at MUST during September and November 2021. We assessed knowledge and practices using an adapted questionnaire. Summary statistics were then used to describe the data.

Results 37 nursing students (64.9\% response rate) participated in our survey. Twenty one (56.8\%) were female and had a modal age range of $20-24$ years. Nineteen (51.4\%) of the students were BNC (Bachelor of Nursing Completion) students, with the rest being BNS (Bachelor of Nursing). More than $75 \%$ of students correctly answered each of two out of the ten questions. More than three quarters of the students reported having done each of six out of the eleven practices surveyed.

Conclusion Nursing students had poor knowledge and fair satisfactory practices regarding monitoring of the fluid requirements in burns patients. We recommend periodic re-trainings and reassessment of clinical skills of nursing students.

\section{Introduction}

Burns present a public health threat causing an estimated 180,000 deaths annually. The majority of these occurring in low- and middle-income countries (Greenhalgh, 2019). Non-fatal burns are a leading cause of morbidity. In 2018, nearly 11 million people were burned severely enough to require medical attention (Burns, 2018). In a sub-Saharan African review, scalds accounting for $59 \%$ of all cases,then direct contact with flames, which were responsible for 33\% (Nthumba, 2016). Despite the high incidence of burns in subSaharan Africa, health care providers skilled in burns care are rare, and there is a need to scale up proper training to manage such patients (Nthumba, 2016). Unfortunately, there is limited literature of nursing students knowledge and understanding of the need to monitor fluid requirement. It is widely accepted that fluid loss is one of the major problems faced following major burns. Appropriate fluid management directly improves the prognosis and outcome of burns patients (Haberal et al., 2010).

According to a scoping review by Mukagaju and colleagues, between 1993 and 2019, 44,369 patients with burns were reported in East Africa. $56 \%$ of these were male. The most common cause of injury was being scalded (61\%), then open flame (17\%) (Mukagaju et al., 2021). Mortality has declined in the past decades but people living in the middle- and low-income countries still face a challenge (Peck, 2011). In East Africa, the mortality rate for burns patients ranges between 0 to $67 \%$ (Mukagaju et al., 
2021). Management involves both immediate care and long-term care. The initial management requires prioritization of the airway, breathing, and circulation, with the goals of long-term care including closure of the wound, management of the hypermetabolic response, and prevention of infection and multiple organ dysfunction (Greenhalgh, 2019).

According to the American Burn Association guidelines, adult and paediatrics patients with burns of greater than $20 \%$ total body surface area (TBSA), should receive fluid resuscitation based on their weight and surface area burnt. (Holaday and McPhearson, $1997 \&$ Moi et al, 2008). A recent experimental study found that a health education program had improved the nurses' knowledge surrounding fluid and electrolyte replacement (Awad, Mohamed, and Hamed, 2020). With poor knowledge around fluid monitoring (Aslam S. et al., 2017), a strong emphasis on fluid balance should become part of training.

Inaccurate monitoring of fluid balance, especially in burns patients, may worsen the patients' condition. Fluids should be monitored and accurately recorded on the ward. This study aimed to examine the knowledge and practices of clinical nursing students at MUST around the monitoring of adult burns' patients in the surgical ward of Mbarara regional referral hospital (MRRH).

\section{Methods}

\section{Study design.}

We conducted an online based cross-sectional study with a quantitative research methods approach.

\section{Study area.}

The study was conducted at Mbarara University of Science and Technology, among nursing students who had rotated on the surgical ward of Mbarara Regional Referral Hospital (MRRH). Mbarara University, is a public university in Uganda. Student intake and instruction commenced in 1989. It is one of the eight public universities and degree-awarding institutions in the country and is accredited by the Uganda National Council of Science and Technology.

\section{Target population}

Bachelor of Nursing students and Bachelor of Nursing completion students.

\section{Study population.}

The study participants included Bachelor of nursing (BNS) and Bachelor of Nursing Completion (BNC) students studying at MUST. BNC and BNS students' study for two and four years respectively at 
MUST and start their clinical years in year one and two respectively. Our targeted population comprised approximately 60 students. They were chosen because they have studied the management of burns, and they have managed burns patients during their clinical studies.

\section{Sample size determination.}

We determined the sample size using Yamane Taro's method (Yamane, 1967:886) of sample size determination which assumes a $95 \%$ level of confidence and a $0.5 p$ value

Yamane's formula,

$\mathrm{n}=\mathrm{N} / 1+\mathrm{N}(\mathrm{e})^{2}$

Where;

$\mathrm{n}=$ sample size

$\mathrm{N}=$ total population which is 60 .

$\mathrm{e}=$ margin of error $=0.05$; the level of precision or level of significance .

$n=60 / 1+60(0.05)^{2}$

$\mathrm{n}=52$ study participants

$10 \%$ compensation for those the researcher may not get (non-correspondence) is 5 participants; from $52+$ $(10 \%$ of 52$)$,

Therefore, the total sample size was 57 participants.

\section{Sampling strategy}

A consecutive sampling technique was used to enrol participants in the study.

\section{Inclusion criteria.}

- Bachelor of Nursing students in the clinical years (years 3 and 4) and the Bachelor of Nursing completion students who have studied burns.

- And students who consented to take part in the study.

\section{Exclusion criteria.}

- Students offline during the data collection period. 


\section{Data collection tools}

The research instrument was composed of a self-administered questionnaire. This was created using Google forms. It consisted of both open and close-ended questions. The questionnaire had three sections A, B, and C (See appendix A). Section A collected students' socio-demographics, while section B (10 questions) and section C (11 questions) consisted of an assessment of the students' knowledge and practices in regards to monitoring of fluid requirements among burn patients respectively. The assessment questions were adapted from a recent study by Awad and colleagues (Awad, Mohamed, and Hamed, 2020).

\section{Scoring system:}

A score was calculated based on the students answers. Each question received a binary answer ( 0 or 1$)$. Scores of greater than $75 \%$ were considered good; Scores between 50 and $75 \%$ were considered average; and scores less than $50 \%$ were considered poor.

We also assessed practical exposure. Each practice question received a binary answer ( 0 or 1$)$, basing on whether students had or had not done a given practice, i.e. $(1)=$ Done, while $(0)=$ Not done. The total practices were considered satisfactory if the total score of a given practice was $\geq 75 \%$, and considered unsatisfactory if it is less than $75 \%$.

\section{Data collection procedure}

Researchers shared a Google form link with the questionnaire to potential participants in their class WhatsApp groups. Digital informed consent was obtained from all participants prior to participation. Data was collected from November 8, 2021 till December 6, 2021 (4 weeks).

\section{Quality control}

We conducted a pilot study on $10 \%$ of the sample size outside the study population (five nursing students from Bishop Stuart University) to ensure that the tool was reliable and valid.

\section{Data management, and analysis}

Data was collected using Google forms, and extracted as an Excel file. Before analysis, it was checked. No duplicates or incomplete entries were found. We used STATA version 14 for analysis. We used descriptive statistics to inform frequency and percentage levels. Analysed data was then presented in the form of tables, and pie charts.

\section{Results}


Overall, we received 37 responses (64.9\% response rate). Most students were aged $20-24$ years and more than half of the students were female $(21,56.7 \%)$ and pursuing BNC $(19,51.4 \%) .64 .9 \%$ of the students surveyed were single. The majority of the students $(23,53.2)$ had spent more than one semester on a surgical ward. (See Table 1)

\section{Knowledge of nursing students regarding fluid monitoring for burns patients.}

Students had insufficient knowledge of fluid monitoring for burns patients. Students demonstrated good knowledge ( $>75 \%$ students correctly answering a given question) for only two out of ten survey questions. $16.2 \%$ of the students were able to correctly state Parklands' formula and $29.7 \%$ of the students knew the importance of fluid replacement during the initial phase of resuscitation. (More details in Table 2)

\section{Practices of nursing students regarding monitoring fluid requirements for burns patients.}

Overall, nursing students had fair practices. Students demonstrated satisfactory practices ( $\geq 75 \%$ students reporting have done a given practice) in six out of eleven practices surveyed. All participants reported performing hand hygiene during caring for burns patients in their fluid replacement stages. $A$ significant big proportion of participants documented any fluids and additives given to patients while on ward. (See Table 3)

\section{Discussion}

This study was designed to assess the knowledge and practices of MUST nursing students around fluid monitoring in hospitalised burns patients on the surgical ward at MRRH. We generally found that nursing students had insufficient knowledge and poor practices. This is the first study looking at the knowledge and practices of nursing students regarding monitoring fluid requirements for adult burns patients. The findings of our study are consistent with pre-intervention findings of prior similar studies (Awad, Mohamed, and Hamed, 2020 and Sheta \& Mahmoud 2018). These found poor knowledge regarding monitoring of fluid replacement for burns patients among nurses prior to enrollment in interventional educational program.

Three quarters of the students managed to pass each of the two out of ten knowledge questions concerning monitoring fluid replacement of burns patient. Only $13.51 \%$ of the participants knew the basic steps in the fluid replacement process. Fluid balance is important in guiding nurses when caring for critically ill patients (Asfour $\mathrm{H}, 2016$ ). Our study participants were students with little experience and exposure to patients. This gap needs addressing as students with insufficient knowledge may cause greater harm.

When looking at fluid administration our study produced results that corroborate previous studies. These found nurses have difficulty in using formulae for fluid replacement (Olszewski et al., 2017 and Awad, Mohamed, and Hamed, 2020). 16.2\% of our study participants were able to correctly state Parklands' formula, while $48.7 \%$ of the students were able to select the right fluid requirement in a set 
scenario using the Parkland formula. Some students may have correctly guessed an answer to the scenario presented to them, producing contradicting results. Interventional studies might address this knowledge gap.

We believe lack of prior training around fluid and electrolyte replacement for burns patients and the limited number of semesters rotated on surgical ward, one semester (14, 37.8\%) and 2 semesters (11, $29.7 \%$ ), may be the cause of lack of knowledge amongst surveyed students.

In contrast, Kanakalakshmi (2014), found more than one third of the working nurses followed safe practices regarding fluid and electrolyte replacement therapy. Our study found a significant proportion of students displaying safe practice regarding fluid replacement for burns patients. More than three quarters of students reported having performed six out of eleven safe practices. There is a need to observe these students and assess to see if the practices were performed correctly.

However, with a small sample size, caution must be taken. Our findings may not be transferable to nursing students at Mbarara University and in Uganda generally.

We were unable to clearly determine the degree of burns which our participants had taken care of.

The aim of this study was to determine the knowledge and practices of nursing students around fluid monitoring in burns patients. Despite its descriptive nature, our investigation has shown that nursing students have poor knowledge and fair practices regarding fluid replacement in this patient cohort. Teaching institutions should have periodic surveys of their students' clinical skills to ensure that they are following appropriate guidelines. In-order to improve the survival rates of burn patients, appropriate fluid management of major burns is crucial.

\section{Strengths and limitations of the study}

Our literature review suggests that this is the first study to address the knowledge and practices for nursing students towards monitoring of fluid replacement in burn patients in Uganda.

With a small sample size, caution must be taken as the findings may not be generalizable to nursing students at Mbarara University or in Uganda generally.

Additionally, we were unable to determine whether students performed the required practices correctly as we subjectively accessed students' skills due to limited resources.

Some students were offline during time of data collection. However, the researchers believe that data from this study will lay a good foundation for future scholars interested in studying the topic.

\section{Conclusion}

The aim of this study was to assess the level of knowledge and the practices of nursing students around fluid replacement in burns patients. Despite its descriptive nature, our investigation has shown that 
nursing students have poor knowledge and fair practices regarding fluid replacement in this patient cohort. There is a need for teaching institutions to survey their students' clinical skills to ensure that they follow the appropriate guidelines in their clinical work.

\section{Recommendations}

Policy makers should advocate for retraining and reassessment of nursing students in the clinical skills acquired.

It would be useful to have supplementary educational programs designed to help hone the clinical skills of nursing students in the area of fluid balance monitoring and replacement.

Further research could determine the efficacy of such programs.

\section{Declarations}

Ethical approval and consent to participate.

The study was performed in accordance with the international ethical standards of the Declaration of Helsinki. The study was ethically approved by the Department of Nursing of Mbarara University of Science and Technology Research Ethics Committee to carry out the study (Ref: DON/ 017). Informed consent was obtained from all participants prior to participation.

\section{Funding}

This work was not funded.

\section{Availability of data and materials}

Available from the corresponding author upon a reasonable request.

\section{Competing interests}

No financial and non-financial interests declared

\section{Author's contributions}

JA developed an idea of the study. JK designed the study. JA administered the survey. JK analysed the data and wrote the first manuscript draft. OG, WM, RK, FL and AT reviewed and edited. All authors read and approved the final manuscript.

\section{Acknowledgements}

None 


\section{Disclosures}

None

\section{References}

Awad, M.O., Mohamed, S.S. and Hamed, S.M. (2020) "Effect of an Educational Program on Nurse's Performance Regard Monitoring Fluid and Electrolyte Replacement for Burned Patients".

Asfour,H. (2016): Fluid Balance Monitoring Accuracy in Intensive Care Units. Alexandria university IOSR Journal of Nursing and Health Science 5(4): 53-62 Available at www. iosrjournals. org Retrieved on11-121019.

Aslam, M., Niazi, M.Z. and Khan, I. (2017) “Epidemiology of paediatric burns at Lady Reading hospital Peshawar". Pak J Surg, 33(1), pp.87-91.

Burns (2018). Available at: https://www.who.int/news-room/fact-sheets/detail/burns (Accessed: 30 June 2021).

Greenhalgh, D.G. (2019) "Management of Burns", New England Journal of Medicine, 380(24), pp. 23492359. doi:10.1056/NEJMra1807442.

Holaday, M. and McPhearson, R.W. (1997) 'Resilience and Severe Burns', Journal of Counseling \& Development, 75(5), pp. 346-356. doi:10.1002/j.1556-6676.1997.tb02350.x.

Kanakalakshmi, R. (2014). Knowledge and practice regarding fluid and electrolytes replacement therapy for patient with burns among working nurses, Narayana Nursing Journal; 3(4): PP. 34-36.

Moi, A.L., Vindenes, H.A. and Gjengedal, E. (2008) "The experience of life after burn injury: a new bodily awareness". Journal of advanced nursing, 64(3), pp.278-286.

Mukagaju, F.,Velin,L. ,Miranda,E.,Shyaka,I.,Nezerwa,Y.,Furaha,C.,Ntirenganya,F.,Riviello,R. and Pompermaier,L. (2021) "What is Known About Burns in East Africa? A Scoping Review", Journal of Surgical Research, 266, pp. 113-124. doi:10.1016/j.jss.2021.03.055.

Nthumba, P.M. (2016) "Burns in sub-Saharan Africa: A review", Burns, 42(2), pp. 258-266. doi:10.1016/j.burns.2015.04.006.

Olszewski, A., Yanes, A., Stafford, J., Greenhalgh, D., Palmieri, T., \& Sen, S. (2017 ). Development and implementation of an innovative burn nursing handbook for quality improvement. J Burn Care Res J; 37(1): PP.20-24.

Peck, M.D. (2011) "Epidemiology of burns throughout the world": Part 1: Distribution and risk factors. Burns, 37(7), pp.1087-1100: 
Sheta, H. \&Mahmoud, M. (2018). Effectiveness of Structured Educational Program on Knowledge and Practice among Nurses Regarding Body Fluid Balance, IOSR Journal of Nursing and Health Science, 7 ( 5):74-83 .

\section{Tables}

Tables 1-3 are in the supplementary files section.

\section{Figures}

\section{Distribution of study participants according to their program of study.}

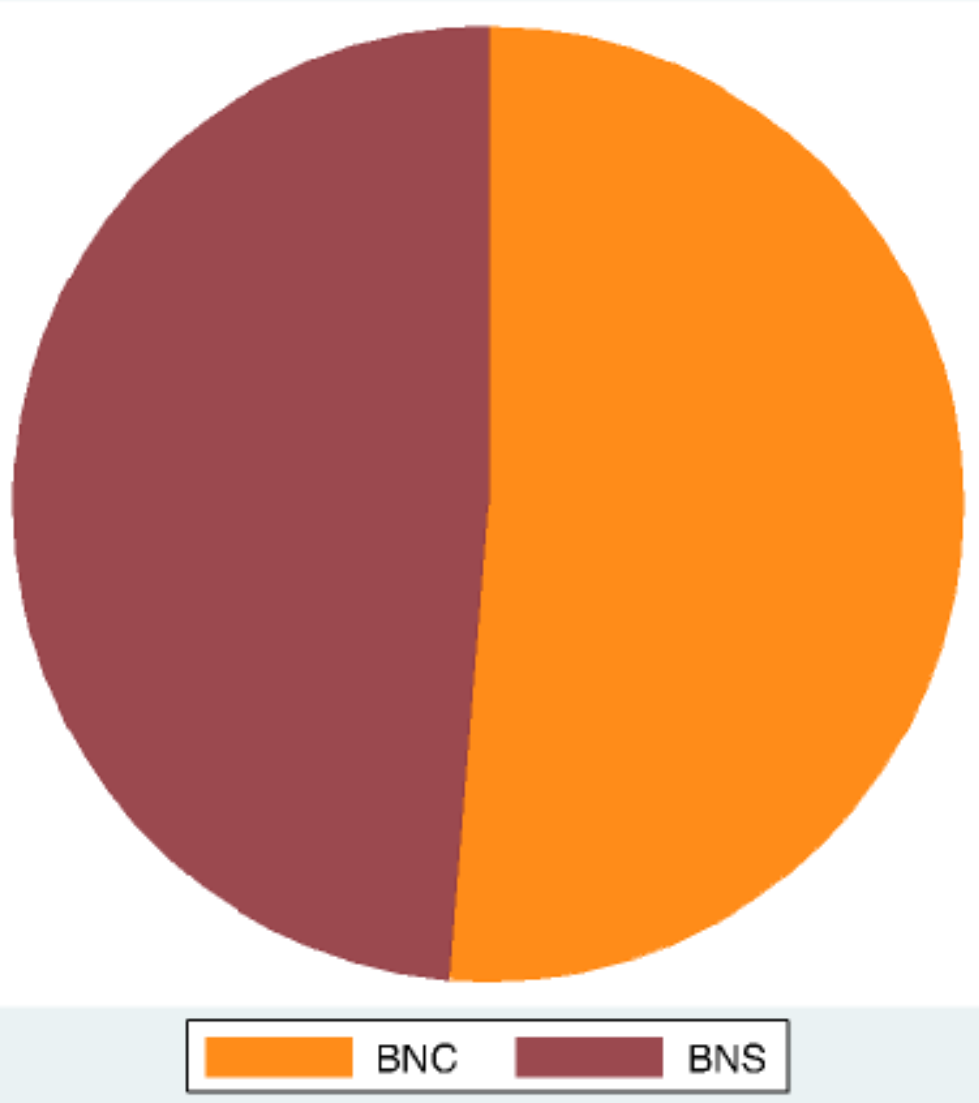

Figure 1

Distribution of study participants according to their program of study. 


\section{Distribution of study participants according to their year of study}

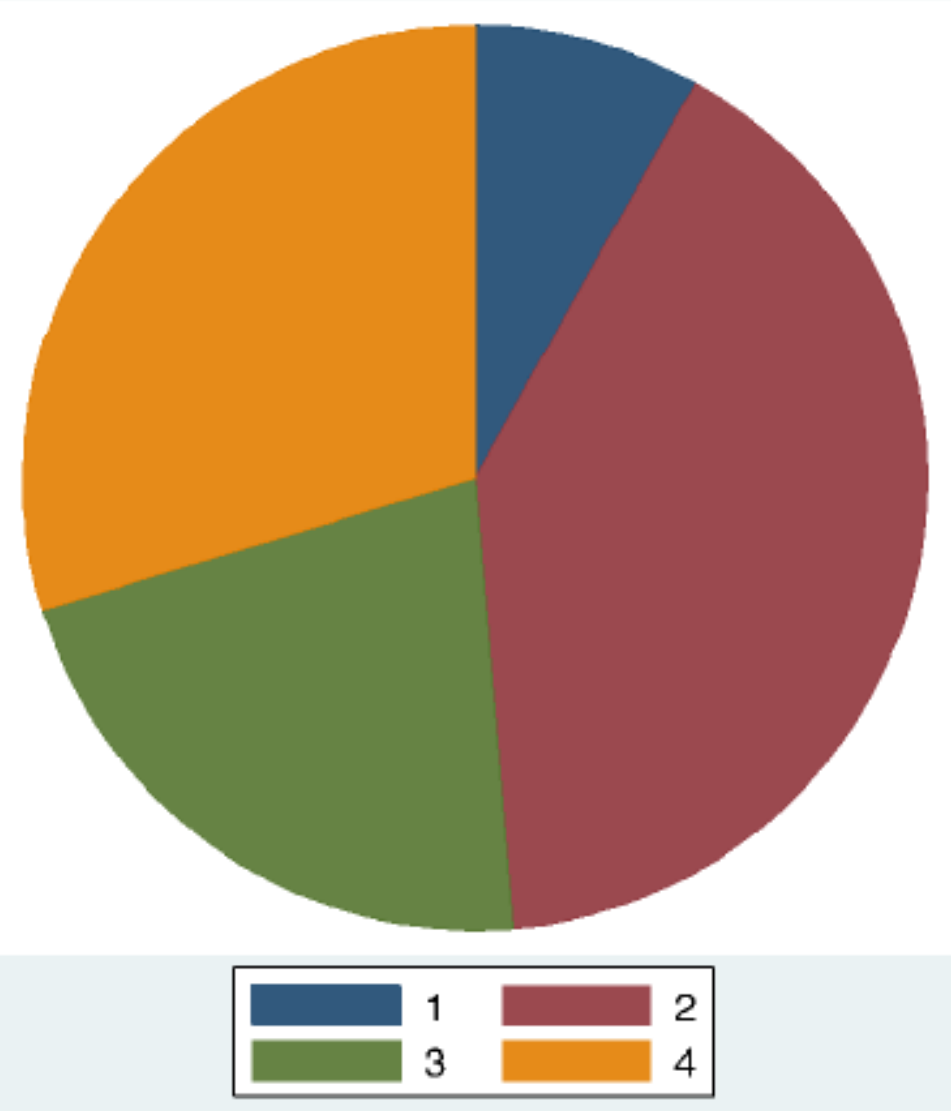

Figure 2

Distribution of study participants according to their year of stud

\section{Supplementary Files}

This is a list of supplementary files associated with this preprint. Click to download.

- Table1.pdf

- Table2.pdf

- Table3.pdf

- Questionnaire.pdf 